Research Article

\title{
Interpretation of Root Canal Anatomy of Maxillary and Mandibular Permanent Canines in Saudi Subpopulation: A Cone-Beam Computed Tomography (CBCT) Study
}

\author{
Amal A. Almohaimede (iD, Alanoud A. Alqahtani, Norah M. Alhatlani, Nouf S. Alsaloom, \\ and Shafia A. Alqahtani \\ Department of Restorative Dental Sciences, Endodontic division, College of Dentistry, King Saud University, P.O. Box 5967, \\ Riyadh 11432, Saudi Arabia \\ Correspondence should be addressed to Amal A. Almohaimede; aalmohaimede@ksu.edu.sa
}

Received 11 January 2021; Revised 28 January 2021; Accepted 29 January 2021; Published 13 February 2021

Academic Editor: Luca Testarelli

Copyright (C) 2021 Amal A. Almohaimede et al. This is an open access article distributed under the Creative Commons Attribution License, which permits unrestricted use, distribution, and reproduction in any medium, provided the original work is properly cited.

\begin{abstract}
The objective of this investigation was to evaluate the root canal anatomy of permanent canines in a Saudi subpopulation utilizing CBCT. A total of 1328 CBCT images of maxillary (634) and mandibular (694) permanent canines were collected from Saudi patients who visited the College of Dentistry at King Saud University in Riyadh. The images were analyzed for root and canal numbers, canal configuration types, and symmetry between the bilateral canine teeth in each arch. Gender, age, and tooth position were recorded. The Kappa test was used for both inter- and intraexaminer reliabilities. Data were analyzed with the chi-square test at a $P$ value $\leq 0.05$. There were no two-rooted teeth in maxillary canines, and $1.89 \%$ had two canals. Double roots and double canals in mandibular canines were found in $2.88 \%$ and $9.94 \%$ of the teeth, respectively. Type I canal configuration was more common in maxillary canines (97.94\%) compared to mandibular canines $(92.07 \%)$. Maxillary canines showed higher bilateral symmetries of root and canal numbers and canal configuration types (100\%, $98.1 \%$, and $97.9 \%$, resp.) compared to mandibular canines (97.1\%, $90.1 \%$, and $92.1 \%$, respectively). The majority of maxillary and mandibular canines had a single root with a single canal and type I canal configuration. Mandibular canines are characterized by having more than one root and canal and alterations in root canal configurations compared to maxillary canines.
\end{abstract}

\section{Introduction}

Awareness of the canal anatomy and its alterations, thorough debridement and shaping of all pulp canals, and complete canal filling are essential steps for favorable root canal treatment [1]. Missing the canal and leaving it without treatment may negatively affect the treatment outcome [2].

Researchers have used various methods and techniques to assess root canal morphology, including serial sectioning $[3,4]$, canal staining and clearing $[5,6]$, conventional and digital radiographic examination [7], and microcomputed tomography $(\mu \mathrm{CT})[8]$. In the late 1990s, three-dimensional scans of the maxillofacial skeleton were developed and since that time, it has become available for dental offices [9]. This method is characterized by a lower radiation dose and shorter acquisition time than conventional computed tomography (CT) [10]. Moreover, cone-beam computed tomography (CBCT) is an accurate and noninvasive method that provides a presentation of the root canal system in three dimensions [11], and its reconstructions showed a very strong correlation with the histologic sections [12].

Several investigators have investigated different variations in root canal morphology $[4,6,13]$. Vertucci classified the root canal morphology into eight types, and many researchers have used it for root canal system classification [6].

Several studies have demonstrated that root canal morphology varies according to race, ethnic group [14-16], and gender $[17,18]$. Therefore, these variations should be acknowledged in the pretreatment analysis for root canal therapy. 
Mandibular and maxillary permanent canines mostly have one root with one canal $[13,19]$. However, several studies have reported different anatomical alterations of maxillary and mandibular permanent canines of different races and genders [20-22].

The objective of this investigation was to evaluate the root canal anatomy of permanent maxillary and mandibular canines in a Saudi subpopulation utilizing CBCT.

\section{Materials and Methods}

The ethics committee at King Saud University, College of Medicine (IRB Project No. E-17-2742), approved this study. A total of 1328 CBCT images of maxillary and mandibular permanent canines were obtained from Saudi patients (565 males and 763 females) aged between 18 and 74 years. These patients attended the Radiology Department at the College of Dentistry at King Saud University in Riyadh between the years 2015 and 2019.

The samples were selected based on the availability of CBCT images of maxillary and/or mandibular canines with full root formation. Excluded images were low-quality CBCT images, previously root canal treated or initiated teeth, presence of root resorption or periapical lesions, presence of coronal or postrestorations, and teeth with immature apices. The total final sample consisting of 634 maxillary canines and 694 mandibular canines was observed and analyzed for root and canal numbers and the configuration types of root canals based on Vertucci's classification [6]. Moreover, the symmetry of roots and canals and the canal configuration between the bilateral canine teeth in each arch were observed and analyzed. Gender, age, and tooth position were reported.

The CBCT images were analyzed at the Radiology Department of the College of Dentistry at King Saud University, by one endodontist and three trained interns for tooth position, root and canal numbers, and canal configuration types. A professional oral radiologist was consulted. The Planmeca Romexis Viewer software was used for image assessment (Planmeca, Roselle IL).

Radiological images were obtained by a professional technician according to the manufacturer's recommended protocol using different CBCT machines: Planmeca ProMax 3D (PLANMECA, Roselle, IL, USA) and CS9300 3D digital imaging system (Carestream, Rochester, NY). The exposure time was $3-15$ seconds. The slice thickness was $0.2 \mathrm{~mm}$ thick viewed from the coronal to apical region, and the voxel size was $75-600 \mu \mathrm{m}$, with small or large fields of view (FOVs) included.

Twenty CBCT images (with 10 maxillary and 10 mandibular canines) were randomly selected according to the evaluation criteria to measure the intra- and interexaminer reliabilities. Images were identified for tooth position, root and canal numbers, and root canal configuration types. For intraexaminer agreement, the same images were reassessed by the same examiner after one week. The interexaminer agreement was measured among the four different examiners.
2.1. Statistical Analysis. For both inter- and intraexaminer reliabilities, the Kappa test was used [23]. For data analysis, chi-square test was used using SPSS 22 software (SPSS Inc, Chicago, IL), and $P$ value $\leq 0.05$ indicated statistical significance.

\section{Results}

For interexaminer reliability, kappa test values were 1 (almost perfect agreement) for the number of roots, 1 (almost perfect agreement) for the number of canals per canal, and 0.8 (substantial agreement) for the configuration of root canals. For intraexaminer reliability, kappa test values were 1 for all examiners regarding root and canal numbers. For root canal configuration types, kappa test values were 1 for the first and fourth examiners, 0.85 for the second examiner, and 0.95 for the third examiner. Kappa test values verified the reliability of the measurements conducted by the four examiners.

The frequency of teeth according to gender and tooth position is summarized in Table 1 .

The number of roots recorded was up to two roots in 20 canines (1.5\%) (Figure 1), and the majority were one-rooted teeth in 1308 canines (98.5\%). The females harbored a greater number of two roots ( 14 canines $1.8 \%)$ than males (6 canines $/ 1.1 \%)$. However, there was no statistically significant difference noted $(P=0.362)$. A significant difference was noted between the number of roots and the tooth position $(P=0.000)$. Of the 694 lower canines, $674(97.11 \%)$ teeth were single-rooted and $20(2.88 \%)$ teeth were double-rooted. However, all the 634 (100\%) maxillary canines had one root.

The number of canines recorded with two canals was 81 $(6.1 \%)$, and the majority of teeth had one canal (1247 canines, 93.9\%). Females harbored a larger number of canines with two canals (55/7.2\%) than males (26/4.6\%). However, no statistically significant difference was noted $(P=0.063)$. A significant difference was noted between the number of canals and the tooth position $(P=0.000)$. Of the 694 mandibular canines, $625(90.05 \%)$ had a single canal, and 69 (9.94\%) had double canals. Of the 634 maxillary canines, 622 (98.1\%) had a single canal, and 12 (1.89\%) had double canals.

Type I canal configuration was mostly observed in 1260 canines (94.9\%), with a statistically significant difference between the other configuration types $(P=0.005)$, followed by Type $\mathrm{V}$ in 24 canines (1.8\%), Type III in 23 canines $(1.7 \%)$, Type II in 14 teeth (1.1\%), Type IV in 5 canines $(0.4 \%)$, and Type VII in two canines $(0.2 \%)$. Regarding gender, Types I, II, III, V, and VII were common in females, whereas Type IV was more frequent in males $(P=0.005)$ (Table 2 ).

A significant relationship was noted between canal configuration and the tooth position $(P=0.003)$. The mandibular canines showed more variations in canal configuration than the maxillary canines. The Type I configuration was more common in maxillary canines (97.94\%) compared to mandibular canines (92.07\%). However, Types II, III, VI, V, and VII were more common in mandibular canines compared to maxillary canines (Table 3 ).

Within the same maxillary arch, both left and right canines existed in 313 patients. In total, 100\% of the teeth 
TABLE 1: The frequency of teeth according to gender and tooth position.

\begin{tabular}{lc}
\hline & Frequency of teeth (\%) \\
\hline Gender & \\
Male & $565(42.5 \%)$ \\
Female & $763(57.5 \%)$ \\
\hline Tooth position & \\
Maxillary right canine & $321(24.2 \%)$ \\
Maxillary left canine & $313(23.6 \%)$ \\
Mandibular left canine & $347(26.1 \%)$ \\
Mandibular right canine & $347(26.1 \%)$ \\
\hline
\end{tabular}

showed symmetrical root numbers, $98.1 \%$ showed symmetrical canal numbers, and $97.9 \%$ showed symmetrical canal configurations. However, in the mandibular arch, both left and right canines existed in 347 patients. In total, 97.1\% of the teeth demonstrated a symmetrical number of roots, $90.1 \%$ showed a symmetrical number of canals, and $92.1 \%$ showed a symmetrical canal configuration.

Regarding age, no difference was observed between the different age groups and the number of roots $(P=0.923)$. However, a statistically significant difference was noted between the different age groups and the number of canals and the type of canal configuration $(P=0.023$ and $P=0.000$, respectively) and the older age group ( $>65$ years old) showed more complicated root canal anatomy. The results are summarized in Tables 4 and 5 .

\section{Discussion}

This study showed anatomical alterations in the morphology of the root canal system of human permanent canines in both arches in a Saudi subpopulation. Dentists must be knowledgeable of the anatomical alterations in the root canal system to avoid iatrogenic procedural errors that arise from inadequate knowledge. Therefore, since 1870, the literature has documented studies on the anatomy of the root canal system of teeth in different populations using different and improved analysis techniques [24, 25]. In this study, the CBCT technique was used to provide a third dimension to analyze the root canal anatomy. The efficiency of CBCT in revealing the morphology of the root canal has been studied and compared with the standard methods in evaluating root canal morphology. Kajan et al. found that CBCT and clearing and staining methods were comparable in detecting the number of root canals of teeth in both arches [26]. Another study showed that CBCT is better than the clearing technique in detecting Type I Vertucci classification [27]. The technique selected to evaluate the anatomy of the root canal should be valid, simple, noninvasive, feasible, and reproducible [28, 29].

The present study investigated the maxillary and mandibular permanent canines' root canal morphologies in a Saudi subpopulation. The incidence of double roots and canals in lower canines in this study was $2.88 \%$ and $9.94 \%$, respectively. Our results are in accordance with the findings of Mashyakhy in his study in a Saudi Arabian population, where he found that $2.7 \%$ of the lower canines had double roots and $9.3 \%$ had double canals [30]. However, the finding of double roots and canals in mandibular canines was less than that reported in a previous study in a Saudi population at $0.2 \%$ and $4.6 \%$, respectively [31]. This difference could be attributed to their smaller sample size. Comparable results to our findings were found in the Turkish population regarding the presence of two-rooted mandibular canines (3.1\%) [32] and the Syrian population (2.15\%) [33]. In Iranian populations, Aminsobhani et al. and Rahimi et al. found higher levels than that noted in our study in mandibular canines with two roots at $4.7 \%$ and $12.08 \%$, respectively [34, 35]. However, our rates are greater than those of Pecora et al. in Brazil (1.7\%) [36], Zhao et al. (0.7\%) in the Chinese population [37], Singh and Pawar in the South Asian Indian population $(0 \%)$ [38], Zhengyan et al. $(0.8 \%)$ in the Chongqing population [39], Pan et al. (1.21\%) in the Malaysian population [40], and Soleymani et al. (1.3\%) in the Iranian population [22]. The presence of two canals in lower canines in other different populations ranged between 0 and $15.1 \%[22,32,34,36,37]$. These morphological variations could be attributed to different ethnic groups.

No two-rooted teeth in maxillary canines were noted in the present study. In total, $98.1 \%$ had one canal, and only $1.89 \%$ had two canals. Our results are consistent with the findings of Mashyakhy in a Saudi population (100\% with one root; $99 \%$ with one canal, and $1 \%$ with two canals) [30]. Additionally, the findings are consistent with those reported in Malaysian and Iranian populations (100\% had one root and one canal in the upper canines in both populations) $[40,41]$. Most of the recorded literature about the presence of two roots in maxillary canines involved case reports [42-44]. This finding indicates that the existence of two roots or canals in maxillary canines is infrequent.

This study showed that Type I canal configuration was found in most mandibular canines $(92.07 \%)$, followed by Type III (2.88\%) and Type V (2.44\%). Our results agree with those reported in a previous investigation in a Saudi subpopulation, where most canal configurations were Type I (90.7\%) followed by Type III (6.1\%) and Type V (3.2\%) [29]. However, another study in a Saudi subpopulation found that most mandibular canines had a Type I canal configuration (95.4\%) followed by Type II (2.6\%) and Type III (1.8\%) [31]. These differences could be explained by the smaller sample size compared to our study. These findings are similar to those reported in several studies in different populations, in which the Type I canal configuration had the highest incidence among other configuration types [22, 33-40] in lower canines.

Concerning maxillary canines, this study showed that the Type I canal configuration was mostly observed (97.94\%) followed by Type V (1.1\%) and Types II and III (0.47\%). Our results are slightly different from what Mashyakhy found in his study in a Saudi Arabian population, where he documented Type I (99\%) and Type III (1\%) canal configurations in maxillary canines [30]. This finding could be explained by the difference in the sample size between both studies. Comparable results were also found among the Turkish population in a study using the clearing technique in maxillary canines, where the Type I canal configuration was 


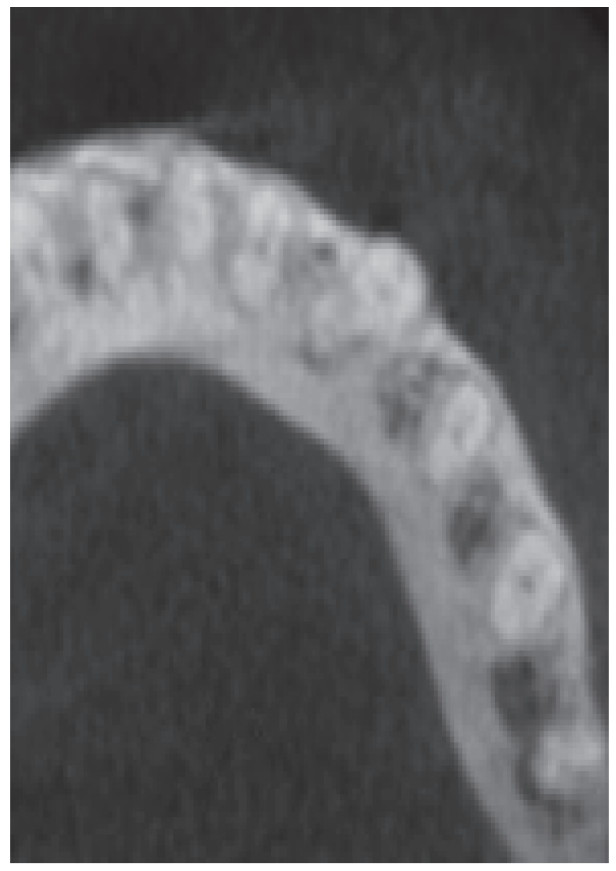

(a)

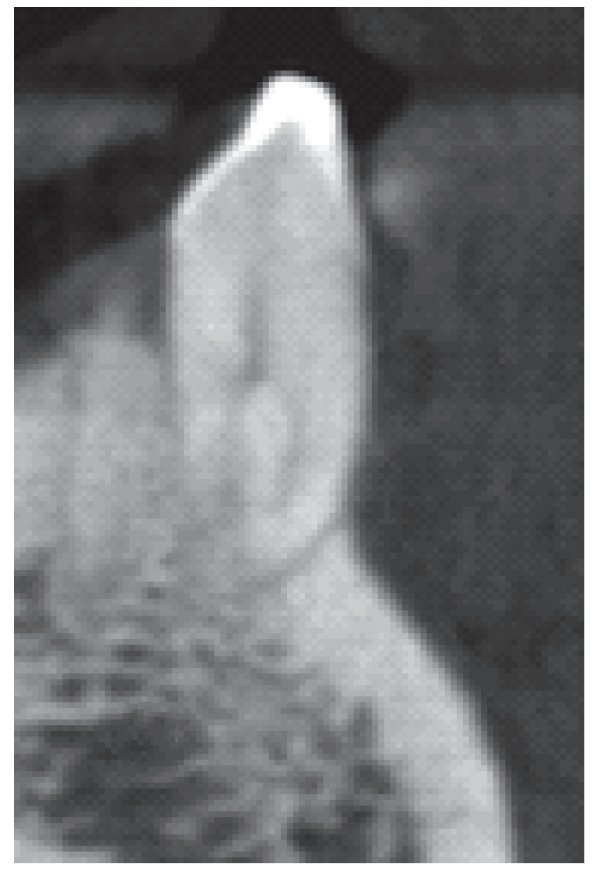

(b)

Figure 1: Cone-beam computed tomography image for mandibular canine with two roots. (a) The axial plane. (b) The coronal plane.

TABLE 2: Variations in canal configuration according to gender.

\begin{tabular}{lcc}
\hline & \multicolumn{2}{c}{ Frequency of teeth (\%) } \\
& Male (565) & Female (763) \\
\hline $\begin{array}{c}\text { Canal configuration type } \\
\text { Type I }\end{array}$ & $542(95.9 \%)$ & $718(94.1 \%)$ \\
Type II & $1(0.2 \%)$ & $13(1.7 \%)$ \\
Type III & $7(1.2 \%)$ & $16(2.1 \%)$ \\
Type IV & $5(0.9 \%)$ & 0 \\
Type V & $10(1.8 \%)$ & $14(1.8 \%)$ \\
Type VI & 0 & 0 \\
Type VII & 0 & $2(0.3 \%)$ \\
Type VIII & 0 & 0 \\
\hline
\end{tabular}

mostly observed (93.48\%) followed by Type III (4.35\%) and Type V (2.17\%) [23]. In contrast, a Type I canal configuration was found in all examined maxillary canines (100\%) in a Malaysian population [40].

Regarding gender, the present study reported no differences in root and canals numbers between genders. However, a significant difference was noted between genders regarding canal configuration $(P=0.005)$. Types I, II, III, V, and VII were more frequent in females than in males, whereas Type IV was more frequent in males than in females. These results could be explained by the larger sample size in females (763) than in males (565). Our findings are in partial agreement with those reported by Mashyakhy in a Saudi Arabian population, where no significance in the root and canal numbers was noted between genders in canines in both arches. Nonetheless, a significant difference was noted between genders regarding canal
TABLE 3: Variations in canal configuration according to tooth position.

\begin{tabular}{lcc}
\hline \multicolumn{3}{c}{ Frequency of teeth (\%) } \\
& Maxillary canines (634) & Mandibular canines (694) \\
\hline Canal configuration type & $639(92.07 \%)$ \\
Type I & $621(97.94 \%)$ & $11(1.58 \%)$ \\
Type II & $3(0.47 \%)$ & $20(2.88 \%)$ \\
Type III & $3(0.47 \%)$ & $5(0.72 \%)$ \\
Type IV & 0 & $17(2.44 \%)$ \\
Type V & $7(1.1 \%)$ & 0 \\
Type VI & 0 & $2(0.28 \%)$ \\
Type VII & 0 & 0 \\
Type VIII & 0 & \\
\hline
\end{tabular}

configurations. Types III and V were more common in females than males, and Type I was more common in males than females [30]. Similarly, in another study in a Saudi population on lower canines, no significant difference was found between gender and the number of roots. However, there was a notable difference between root canal configuration and gender. Types II, III, and IV canal configurations were more frequent in males and Type I was more frequent in females [31]. In a Malaysian subpopulation, similar results were reported regarding the absence of differences between genders in the number of roots and canals [40]. In contrast to our results, in an Iranian population, males have a higher incidence of double roots and canals in lower canines [22]. On the other hand, Martins et al. reported that females had more root numbers than males in lower canines in a Portuguese population [18]. 
TABle 4: Prevalence of root and canal number among different age groups.

\begin{tabular}{lcccc}
\hline \multirow{2}{*}{ Age range groups (years old) } & \multicolumn{2}{c}{ Prevalence of teeth } & \multicolumn{2}{c}{ Prevalence of teeth } \\
& With one root (\%) & With two roots (\%) & With one canal (\%) & With two canals (\%) \\
\hline $18-23$ & 98 & 2 & 94.1 & 5.9 \\
$24-29$ & 99.2 & 0.8 & 93.8 & 6.2 \\
$30-35$ & 98.6 & 1.4 & 96.4 & 3.6 \\
$36-41$ & 97.6 & 2.4 & 88.1 & 11.9 \\
$42-47$ & 98.8 & 1.2 & 97.6 & 2.4 \\
$48-53$ & 98.1 & 1.9 & 91.7 & 8.3 \\
$54-59$ & 98.7 & 1.3 & 98.7 & 92.3 \\
$60-65$ & 96.9 & 3.1 & $85.7 *$ & 7.7 \\
$>65$ & 100 & 0 & $85.3 *$ \\
\hline
\end{tabular}

* Significant at $P \leq 0.05$.

Table 5: Prevalence of different types of canal configurations among different age groups.

\begin{tabular}{|c|c|c|c|c|c|c|}
\hline \multirow{2}{*}{ Age range groups (years old) } & \multicolumn{6}{|c|}{ Canal configurations } \\
\hline & Type I & Type II & Type III & Type IV & Type V & Type VII \\
\hline $18-23$ & $94.9 \%$ & $0.4 \%$ & $0.8 \%$ & $1.2 \%$ & $2.8 \%$ & 0 \\
\hline $24-29$ & $94.6 \%$ & $1.2 \%$ & $2.5 \%$ & 0 & $1.7 \%$ & 0 \\
\hline $30-35$ & $97.1 \%$ & 0 & $2.1 \%$ & 0 & $0.7 \%$ & 0 \\
\hline $36-41$ & $89.7 \%$ & $0.8 \%$ & $4.8 \%$ & 0 & $4.8 \%$ & 0 \\
\hline $42-47$ & $98.8 \%$ & $1.2 \%$ & 0 & 0 & 0 & 0 \\
\hline $48-53$ & $91.7 \%$ & $1.9 \%$ & $1.9 \%$ & 0 & $4.6 \%$ & 0 \\
\hline $54-59$ & $100 \%$ & 0 & 0 & 0 & 0 & 0 \\
\hline $60-65$ & $95.4 \%$ & 0 & 0 & $1.5 \%$ & 0 & $3.1 \%$ \\
\hline$>65$ & $85.7 \% *$ & $3.6 \% *$ & $7.1 \% *$ & 0 & $3.6 \%$ & 0 \\
\hline
\end{tabular}

* Significant at $P \leq 0.05$.

The current study examined bilateral symmetry in canines in both arches. In lower canines, our results showed high bilateral symmetry for the root numbers (97.1\%), number of canals (90.1\%), and canal configuration (92.1\%). These results agree with the findings of Mashyakhy ( $95.5 \%$ for root numbers, 91.1\% for canal numbers, and $90.1 \%$ for canal configuration) [30] and Al-Dahman et al. (97.7\% for the root numbers and canal configuration) [31] in a Saudi Arabian population. Our results are also consistent with that found in an Iranian population, where Soleymani et al. reported $95.4 \%$ bilateral symmetry in root numbers and canal configuration in lower canines [22]. In contrast, Kayaoglu et al. reported 28\% bilateral symmetry in canal numbers in mandibular canines in a Turkish population [32].

Our results in maxillary canines showed higher bilateral symmetry than mandibular canines for roots (100\%), canals (98.1\%), and canal configuration (97.9\%). This finding was consistent with Mashyakhy's study in a Saudi population (100\% for root numbers, $98.9 \%$ for canal numbers, and 98.9\% for canal configuration) [30]. Karataslioglu and Kalabalik reported in their study in a Turkish population that $96.6 \%$ of the maxillary canines had bilateral symmetry in the root canal configuration [45].

The effect of age on tooth anatomy has rarely been studied. In our study, the results showed that the older age group ( $>65$ years old) had a higher frequency of two canals than the younger age groups and a more complex root canal configuration. These results can be clarified by the physiological change in root canal system morphology during aging through the formation of secondary dentine. Similar results were found by Karataslioglu and Kalabalik in a Turkish population [45] and by Martins et al. in a study on the effect of age on the root canal system configuration using CBCT [46]. In contrast, previous studies in Turkish and Chongqing populations showed that younger patients had more frequent multiple canals detected than older patients $[32,39]$.

The data of the current study were limited to one place, where the images were analyzed from one center in one region with variations in the number of samples among the different age groups and gender. Therefore, future studies are recommended to include different regions with different centers with increasing the sample sizes.

\section{Conclusion}

Within the limitations of this study in a Saudi subpopulation, the majority of canines in both arches were singlerooted with single canal and Type I canal configuration. Lower canines demonstrated a higher incidence of double roots and canals than upper canines with more variations in canal configurations. The older age group ( $>65$ years old) showed more complex root canal anatomy. Lower canines showed higher asymmetries in the number of roots and canals and in the type of canal configuration compared to upper canines. Therefore, clinicians need to consider these morphological variations in the pretreatment analysis for the root canal therapy. 


\section{Data Availability}

The data used to support the findings of this study are available from the corresponding author upon request.

\section{Conflicts of Interest}

The authors declare that there are no conflicts of interest.

\section{References}

[1] F. J. Vertucci, "Root canal morphology and its relationship to endodontic procedures," Endodontic Topics, vol. 10, no. 1, pp. 3-29, 2005.

[2] M. D. Peikoff and J. R. Trott, "An endodontic failure caused by an unusual anatomical anomaly," Journal of Endodontics, vol. 3, no. 9, pp. 356-359, 1977.

[3] J. C. Kulild and D. D. Peters, "Incidence and configuration of canal systems in the mesiobuccal root of maxillary first and second molars," Journal of Endodontics, vol. 16, no. 7, pp. 311-317, 1990.

[4] F. S. Weine, H. J. Healey, H. Gerstein, and L. Evanson, "Canal configuration in the mesiobuccal root of the maxillary first molar and its endodontic significance," Oral Surgery, Oral Medicine, Oral Pathology, vol. 28, no. 3, pp. 419-425, 1969.

[5] X.-L. Weng, S.-B. Yu, S.-L. Zhao et al., "Root canal morphology of permanent maxillary teeth in the Han nationality in Chinese Guanzhong area: a new modified root canal staining technique," Journal of Endodontics, vol. 35, no. 5, pp. 651-656, 2009.

[6] F. J. Vertucci, "Root canal anatomy of the human permanent teeth," Oral Surgery, Oral Medicine, Oral Pathology, vol. 58, no. 5, pp. 589-599, 1984.

[7] Y. Sun, T.-Y. Lu, Y. C. Chen, and S.-F. Yang, “The best radiographic method for determining root canal morphology in mandibular first premolars: a study of Chinese descendants in Taiwan," Journal of Dental Sciences, vol. 11, no. 2, pp. 175-181, 2016.

[8] W. Alkaabi, E. Alshwaimi, I. Farooq, H. E. Goodis, and S. M. A. Chogle, "A micro-computed tomography study of the root canal morphology of mandibular first premolars in an Emirati population," Medical Principles and Practice, vol. 26, no. 2, pp. 118-124, 2017.

[9] S. Patel, "New dimensions in endodontic imaging: Part 2. Cone beam computed tomography," International Endodontic Journal, vol. 42, no. 6, pp. 463-475, 2009.

[10] Y. Arai, K. Honda, K. Iwai, and K. Shinoda, "Practical model "3DX" of limited cone-beam X-ray CT for dental use," International Congress Series, vol. 1230, pp. 713-718, 2001.

[11] A. Mazzoni, A. Pacifici, A. Zanza et al., "Assessment of realtime operative torque during nickel-titanium instrumentation with different lubricants," Applied Sciences, vol. 10, no. 18, p. $6201,2020$.

[12] J. Michetti, D. Maret, J.-P. Mallet, and F. Diemer, "Validation of cone beam computed tomography as a tool to explore root canal anatomy," Journal of Endodontics, vol. 36, no. 7, pp. 1187-1190, 2010.

[13] F. Pineda and Y. Kuttler, "Mesiodistal and buccolingual roentgenographic investigation of 7,275 root canals," Oral Surgery, Oral Medicine, Oral Pathology, vol. 33, no. 1, pp. 101-110, 1972.

[14] J. N. R. Martins, Y. Gu, D. Marques, H. Francisco, and J. Carames, "Differences on the root and root canal morphologies between asian and white ethnic groups analyzed by cone-beam computed tomography," Journal of Endodontics, vol. 44, no. 7, pp. 1096-1104, 2018.

[15] P. U. Deng, M. S. Halim, S. A. M. Masudi, S. Al-Shehadat, and B. Ahmad, "Cone-beam computed tomography analysis on root and canal morphology of mandibular first permanent molar among multiracial population in East Coast Malaysian population," European Journal of Dentistry, vol. 12, no. 03, pp. 410-416, 2018.

[16] J. Kottoor, D. Albuquerque, N. Velmurugan, and J. Kuruvilla, "Root anatomy and root canal configuration of human permanent mandibular premolars: a systematic review," Anatomy Research International, vol. 2013, Article ID 254250, 14 pages, 2013.

[17] M. Mashyakhy and G. Gambarini, "Root and root canal morphology differences between genders: a comprehensive in-vivo CBCT study in a Saudi population," Acta Stomatologica Croatica, vol. 53, no. 3, pp. 213-246, 2019.

[18] J. N. R. Martins, D. Marques, H. Francisco, and J. Caramês, "Gender influence on the number of roots and root canal system configuration in human permanent teeth of a Portuguese subpopulation," Quintessence International (Berlin, Germany: 1985), vol. 49, no. 2, pp. 103-111, 2018.

[19] R. Ouellet, "Mandibular permanent cuspids with two roots," Journal of the Canadian Dental Association, vol. 61, no. 2, pp. 159-61, 1995.

[20] N. S. Amardeep, S. Raghu, and V. Natanasabapathy, "Root canal morphology of permanent maxillary and mandibular canines in indian population using cone beam computed tomography," Anatomy Research International, vol. 2014, Article ID 731859, 7 pages, 2014.

[21] M. A. Versiani, J. D. Pecora, and M. D. Sousa-Neto, "Microcomputed tomography analysis of the root canal morphology of single-rooted mandibular canines," International Endodontic Journal, vol. 46, no. 9, pp. 800-807, 2013.

[22] A. Soleymani, N. Namaryan, E. Moudi, and A. Gholinia, "Root canal morphology of mandibular canine in an Iranian population: a CBCT assessment," Iranian Endodontic Journal, vol. 12, no. 1, pp. 78-82, 2017.

[23] J. R. Landis and G. G. Koch, "The measurement of observer agreement for categorical data," Biometrics, vol. 33, no. 1, pp. 159-174, 1977.

[24] M. K. Ataliaykan, Y. Pehlivan, F. Sepetasioaylu, M. Tulirkul̀in, and S. S. Tuncer, "Root canal morphology of human permanent teeth in a Turkish population," Journal of Endodontics, vol. 21, no. 4, pp. 200-204, 1995.

[25] G. Miccoli, A. Cicconetti, G. Gambarini et al., "A new device to test the bending resistance of mechanical endodontic instruments," Applied Sciences, vol. 10, no. 20, pp. 1-6, 2020.

[26] Z. D. Kajan, M. Taramsari, N. Khosravi Fard, and M. Kanani, "Accuracy of cone-beam computed tomography in comparison with standard method in evaluating root canal morphology: an in vitro study," Iranian Endodontic Journal, vol. 13, no. 2, pp. 181-187, 2018.

[27] R. Ordinola-Zapata, C. M. Bramante, M. A. Versiani et al., "Comparative accuracy of the Clearing Technique, CBCT and Micro-CT methods in studying the mesial root canal configuration of mandibular first molars," International Endodontic Journal, vol. 50, no. 1, pp. 90-96, 2017.

[28] P. Neelakantan, C. Subbarao, and C. V. Subbarao, "Comparative evaluation of modified canal staining and clearing technique, cone-beam computed tomography, peripheral quantitative computed tomography, spiral computed tomography, and plain and contrast medium-enhanced digital radiography in studying root canal morphology," Journal of Endodontics, vol. 36, no. 9, pp. 1547-1551, 2010. 
[29] R. Zhang, H. Wang, Y.-Y. Tian, X. Yu, T. Hu, and P. M. H. Dummer, "Use of cone-beam computed tomography to evaluate root and canal morphology of mandibular molars in Chinese individuals," International Endodontic Journal, vol. 44, no. 11, pp. 990-999, 2011.

[30] M. Mashyakhy, "Prevalence of a second root and canal in mandibular and maxillary canines in a Saudi Arabian population: a cone-beam computed tomography study," The Journal of Contemporary Dental Practice, vol. 20, no. 7, pp. 773-777, 2019.

[31] Y. Al-Dahman, A. Alqedairi, H. Alfawaz, F. Alnassar, and A. Al-Jebaly, "Cone-beam computed tomographic evaluation of root canal morphology of mandibular canines in a Saudi subpopulation," Saudi Endodontic Journal, vol. 9, no. 2, pp. 113-118, 2019.

[32] G. Kayaoglu, I. Peker, M. Gumusok, C. Sarikir, A. Kayadugun, and O. Ucok, "Root and canal symmetry in the mandibular anterior teeth of patients attending a dental clinic: CBCT study," Brazilian Oral Research, vol. 29, no. 1, pp. 1-7, 2015.

[33] M. Doumani, A. Habib, A. Alhalak, T. Al-Nahlawi, F. Al Hussain, and S. Alanazi, "Root canal morphology of mandibular canines in the Syrian population: a CBCT assessment," Journal of Family Medicine and Primary Care, vol. 9, no. 2, pp. 552-555, 2020.

[34] M. Aminsobhani, M. Sadegh, N. Meraji, H. Razmi, and M. J. Kharazifard, "Evaluation of the root and canal morphology of mandibular permanent anterior teeth in an Iranian population by cone-beam computed tomography," Journal of Dentistry (Tehran), vol. 10, no. 4, pp. 358-366, 2013.

[35] S. Rahimi, A. S. Milani, S. Shahi, Y. Sergiz, S. Nezafati, and M. Lotfi, "Prevalence of two root canals in human mandibular anterior teeth in an Iranian population," Indian Journal of Dental Research, vol. 24, no. 2, pp. 234-236, 2013.

[36] J. D. Pécora, M. D. Sousa Neto, and P. C. Saquy, "Internal anatomy, direction and number of roots and size of human mandibular canines," Brazilian Dental Journal, vol. 4, no. 1, pp. 53-57, 1993.

[37] Y. Zhao, Y. Dong, X. Wang et al., "Cone-beam computed tomography analysis of root canal configuration of 4674 mandibular anterior teeth beijing da xue xue bao yi xue," Ban, vol. 46, no. 1, pp. 95-99, 2014.

[38] S. Singh and M. Pawar, "Root and canal morphology of mandibular incisors and canines in South Asian Indian population by canal staining and tooth clearing technique," Endodontology, vol. 28, no. 2, pp. 148-153, 2016.

[39] Y. Zhengyan, L. Keke, W. Fei, L. Yueheng, and Z. Zhi, "Conebeam computed tomography study of the root and canal morphology of mandibular permanent anterior teeth in a Chongqing population," Therapeutics and Clinical Risk Management, vol. 12, pp. 19-25, 2016.

[40] J. Y. Y. Pan, A. Parolia, S. R. Chuah, S. Bhatia, S. Mutalik, and A. Pau, "Root canal morphology of permanent teeth in a Malaysian subpopulation using cone-beam computed tomography," BMC Oral Health, vol. 19, no. 1, p. 14, 2019.

[41] M. Kuzekanani and A. M. Jafari, "Root canal anatomy and morphology of permanent maxillary canine teeth in an Iranian population," Italian Journal of Anatomy and Embryology, vol. 124, no. 3, pp. 403-408, 2019.

[42] N. Bola and S. R. Kavuri, "A case of unusual root morphology: maxillary canine with two roots," Journal of the International Clinical Dental Research Organisation, vol. 1, no. 3, pp. 70-75, 2009.
[43] M. I. Weisman, "A rare occurrence: a bi-rooted upper canine," Australian Endodontic Journal, vol. 26, no. 3, pp. 119-120, 2000.

[44] R. Barkhordar and N. Nguyen, "Maxillary canine with two roots," Journal of Endodontics, vol. 11, no. 5, pp. 224-227, 1985.

[45] E. Karataslioglu and F. Kalabalik, "Morphological evaluation of maxillary and mandibular canines using cone-beam computed tomography in Turkish population," Annals of Medical Research, vol. 26, no. 10, pp. 2312-2319, 2019.

[46] J. N. R. Martins, R. Ordinola-Zapata, D. Marques, H. Francisco, and J. Caramês, "Differences in root canal system configuration in human permanent teeth within different age groups," International Endodontic Journal, vol. 51, no. 8, pp. 931-941, 2018. 